Pacific Journal of Mathematics

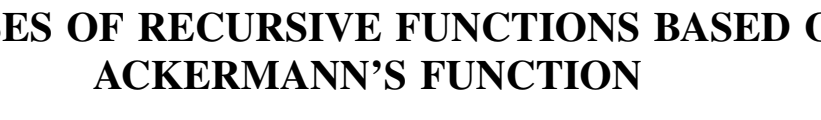




\title{
CLASSES OF RECURSIVE FUNCTIONS BASED ON ACKERMANN'S FUNCTION
}

\author{
ROBERT W. RITCHIE
}

\begin{abstract}
Grzegorczyk has defined an increasing sequence of classes $\mathscr{E}^{n}$ of functions with the properties that $\mathscr{E}^{3}$ is the class of elementary functions of Csillag-Kalmar and $\cup \mathscr{E}^{n}$ is the class of primitive recursive functions. Further, $\mathscr{E}^{n+1}$ properly contains $\mathscr{E}$, if $n>2$ then $\mathscr{E}{ }^{n+1}$ contains a "universal function" over all one-argument functions in $\mathscr{E}^{n}$, and a sequence of functions $g_{n}(x, y)$ in terms of which the $\mathscr{E}^{n}$ are defined has the property that each $g_{n+1}(x, x)$ (eventually) majorizes all the one-argument functions in $\mathscr{E}^{n}$.

The functions $g_{n}(x, y)$ are defined by somewhat artificial nested recursions, and Grzegorczyk poses the following question: "Can the same theorems be proved for classes $\mathscr{F}^{n}$ as for the classes $\mathscr{E}^{n}$ ?" Here $\mathscr{F}^{n}$ differs from $\mathscr{E}^{n}$ only in substituting a more natural function $f_{n}^{\prime}(x, y)$ for each $g_{n}(x, y)$ in the definition of the class. In this paper, we answer his question affirmatively. Indeed, we prove that $\mathscr{F}^{n}=\mathscr{E}^{n}$ for all $n \geqq 0$, and further, $f_{n+1}^{\prime}(x, x)$ eventually majorizes all the one-argument functions in $\mathscr{F}^{n}$.
\end{abstract}

In the first section below, we define the functions $f_{n}(x, y)$ (trivial variants of Grzegorczyk's $\left.f_{n}^{\prime}(x, y)\right)$ which we shall use in place of $g_{n}(x, y)$, and develop various properties of these functions. In the second section, we define and study a sequence $\mathscr{G}_{n}$ of classes of one-argument functions. Each $\mathscr{G}_{n}$ is defined from $f_{n}(x, x)$ and other initial functions by composition and pure iteration as studied by Robinson [7]. In the third section, we define $\mathscr{F}_{n}$ (slight modifications of Grzegorczyk's $\mathscr{F}^{n}$ ) and establish various properties of these classes including that $\cup \mathscr{F}_{n}$ is the class of all primitive recursive functions. In the final section we establish the equality of $\mathscr{F}_{n}$ and $\mathscr{E}^{n}$, and then discuss Grzegorczyk's $f_{n}^{\prime}(x, y)$ and prove that $\mathscr{F}^{n}$ also equals $\mathscr{E}^{n}$.

1. The functions $f_{n}(x, y)$. In [1], Ackermann defines a function of three variables which he shows is not primitive recursive. He obtains this function by considering the functions $x+y, x y$ and $x^{y}$, observing that each is obtained from the preceding by a recursive definition and generalizing this process. Let us depart slightly from [1] and generalize the sequence of three functions as follows:

Received January 25, 1964. This work was supported by National Science Foundation grant GP-1851. 
Definition 1.1. For each $n \geqq 0$, define the function $f_{n}(x, y)$ by

$$
\left.\begin{array}{rl}
f_{0}(x, y) & =x+1 \\
f_{1}(x, y) & =x+y \\
f_{2}(x, y) & =x \cdot y \\
f_{n+1}(x, 0) & =1 \\
f_{n+1}(x, y+1) & =f_{n}\left(x, f_{n+1}(x, y)\right)
\end{array}\right\}
$$

for all $n \geqq 2$.

This differs from Ackermann's generalization in that we begin with $x+1$, not $x+y$, and also we set $f_{n}(x, 0)$ equal to 1 instead of $x$ for all $n \geqq 4$. The resulting difference between $f_{n}(x, y)$ and $\varphi(x, y, n)$ as defined in [1, p. 120] (or $\xi(n, y, x)$ as the function is written in [5, p. 272]) is minor as the following, which results from Lemma 1.1, shows

$$
\begin{array}{r}
f_{n+1}(x, y)=\varphi(x, y, n)=\xi(n, y, x) \text { for } n=0,1,2, \text { all } x, y ; \\
f_{n+1}(x, y+1) \leqq \varphi(x, y, n)=\xi(n, y, x)<f_{n+1}(x, y+2) \\
\text { for } n \geqq 3, x \geqq 2, \text { all } y .
\end{array}
$$

These changes from Ackermann's definition were made here because starting with $f_{0}(x, y)=x+1$ aligns the subscripts or our functions with those of Grzegorczyk, and letting $f_{n}(x, 0)$ be 1 instead of $x$ simply seems more natural to the author.

Let us begin our study of the functions $f_{n}(x, y)$ by establishing three lemmas which yield information on the rate of growth of these functions. These three lemmas are closely related to Ackermann's results (properties III and IIIB, IV, and V of [1, pp. 121-2]).

LEMmA 1.1 . For all $n \geqq 1, x \geqq 2$, and $y \geqq 0, f_{n}(x, y+1)>f_{n}(x, y) \geqq$ $y+1$. Hence $f_{n}(x, z)>f_{n}(x, y) \geqq y+1$ for all $z>y$.

Proof. For $n=1$ this simply states that $x+y+1>x+y \geqq$ $y+1$. Let us assume that the result is true for $n$ and establish its validity for $n+1$. Since $f_{n+1}(x, 1)=x>f_{n+1}(x, 0)=1$, the result holds for $y=0$. Let us prove it for all $y$ by induction. Assume $f_{n+1}(x, y+1)>f_{n+1}(x, y) \geqq y+1$; then $f_{n+1}(x, y+2)=f_{n}\left(x, f_{n+1}(x, y+\right.$ $1)) \geqq f_{n+1}(x, y+1)+1>f_{n+1}(x, y+1)$ by the assumed validity of the theorem at $n$. Further $f_{n+1}(x, y+1)>y+1$, or $f_{n+1}(x, y+1) \geqq y+2$ by the assumption immediately above.

LemMA 1.2. For all $n \geqq 0, x \geqq 1$, and $y \geqq 1, f_{n}(x, y)<f_{n}(x+$ $1, y)$; hence $f_{n}(x, y)<f_{n}(z, y)$ for all $z>x$.

Proof. Immediate for $n=0,1$ and 2. Let us now assume that we have established the theorem for $n$, and show then that it holds for $n+1$. Since for all $y f_{n+1}(1, y)=1$ and $f_{n+1}(2, y) \leqq 2$ (using 
$f_{n+1}(2,1)=2$ and Lemma 1.1), we have the result if $x=0$ or $x=1$. Thus let us assume $x \geqq 2$. Now $f_{n+1}(x, 1)=x<x+1=f_{n+1}(x+1,1)$ so the conclusion holds for $y=1$. Assume it for $y$. Then it also holds for $y+1$ since

$$
\begin{aligned}
f_{n+1}(x+1, y+1) & =f_{n}\left(x+1, f_{n+1}(x+1, y)\right) \\
& >f_{n}\left(x, f_{n+1}(x+1, y)\right) \\
& >f_{n}\left(x, f_{n+1}(x, y)\right) \\
& =f_{n+1}(x, y+1) .
\end{aligned}
$$

Here we used the assumption of the lemma at $n$ and then the assumption for $y$ at $n+1$ together with Lemma 1.1. This completes the proof.

LEMmA 1.3. For all $n \geqq 2, x \geqq 2, y \geqq 0 ; f_{n}(x, y) \leqq f_{n+1}(x, y)$.

$$
\text { Proof. } \begin{aligned}
f_{n+1}(x, 0)=1 & \geqq f_{n}(x, 0) \text { for all } n \geqq 2 \\
f_{n+1}(x, y+1) & =f_{n}\left(x, f_{n+1}(x, y)\right) \\
& \geqq f_{n}(x, y+1) .
\end{aligned}
$$

The last step follows since $f_{n+1}(x, y) \geqq y+1$ and $f_{n}(x, z) \geqq f_{n}(x, y+1)$ for any $z \geqq y+1$, both by Lemma 1.1 .

These three lemmas will be used freely in the work below. Rather than refer to them explicitly, we shall often simply refer to the "monotonicity properties" of the $f_{n}(x, y)$.

We need one further important property of the functions $f_{n}(x, y)$. In Theorems 2.2 and 2.3 in the next section, we shall need to know that $f_{n}\left(f_{n}(x, k), l\right) \leqq f_{n}(x, m)$ for some $m$ dependent only upon $k$ and $l$ whenever $x$ is sufficiently large. In Theorem 1.1 below we find that we can take $m=f_{n-1}(k, l)$. The proof of this comprises the next five lemmas and we get the companion result

$$
f_{n-1}\left(f_{m}(x, y), f_{m}(x, z)\right) \leqq f_{m}(x, y+z)
$$

for all $m \geqq n$ automatically in the proof. The remainder of this section is devoted exclusively to the sequence of lemmas leading to the proof of Theorem 1.1.

Lemma 1.4. For all $m \geqq 3$ and all $x, y$, and $z$,

$$
f_{2}\left(f_{m}(x, y), f_{m}(x, z)\right) \leqq f_{m}(x, y+z) \text {. }
$$

Proof by induction on $m$. In the case $m=3$, the lemma is immediate since $f_{3}(x, y)=x^{y}$, and $f_{2}(x, y)$ is multiplication of $x$ by $y$. Let us assume that the lemma is true for a fixed $m$ (and for all $x, y$, and 
z). Under this assumption we shall prove that $f_{m+1}(x, y) \cdot f_{m+1}(x, z) \leqq$ $f_{m+1}(x, y+z)$ for all $(y, z)$ with $y \leqq z$ which suffices by symmetry.

First we note that since $f_{m+1}(x, 0)=1$, we have $f_{m+1}(x, 0) \cdot f_{m+1}(x, z) \leqq$ $f_{m+1}(x, 0+z)$ for all $z \geqq 0$. We now proceed by induction on $y$, assuming that we know the result for some $y$ and all $z^{\prime} \geqq y$ and proving it for $y+1$ and all $z \geqq y+1$. We begin this verification by noting that the cases $x=0$ and $x=1$ satisfy the desired inequality since $f_{m+1}(0, y)$ is 0 or 1 as $y$ is odd or even, and $f_{m+1}(1, y)=1$ for all $y$, so that we may assume $x \geqq 2$. Consider a value of $z \geqq y+1>0$,

$$
\begin{aligned}
f_{m+1}(x, y & +1) \cdot f_{m+1}(x, z) \\
& =f_{m}\left(x, f_{m+1}(x, y)\right) \cdot f_{m}\left(x, f_{m+1}(x, z-1)\right) \\
& \leqq f_{m}\left[x, f_{m+1}(x, y)+f_{m+1}(x, z-1)\right] \\
& \leqq f_{m}\left[x, f_{m+1}(x, y) \cdot f_{m+1}(x, z)\right] \\
& \leqq f_{m}\left(x, f_{m+1}(x, y+z)\right) \\
& =f_{m+1}(x,(y+1)+z) .
\end{aligned}
$$

Here we have used, in order, the assumption of the lemma for the fixed value $m$, the fact that $a+b \leqq a(b+1)$ for all $a, b \geqq 1$ along with the monotonicity of $f_{m+1}(x, z)$ to see that $f_{m+1}(x, y)+f_{m+1}(x, z-1) \leqq$ $f_{m+1}(x, y) \cdot f_{m+1}(x, z)$, the monotonicity of $f_{m}(x, w)$, and finally the assumption that $f_{m+1}(x, y) \cdot f_{m+1}(x, z) \leqq f_{m+1}(x, y+z)$ for our fixed value of $y$ and any larger $z$.

Lemma 1.5 . For all $x \geqq 2, k \geqq 0, l \geqq 0 ; f_{3}\left(f_{3}(x, k), l\right)=f_{3}(x, k \cdot l)$.

We omit the straightforward inductive proof of this lemma since it merely states that $\left(x^{k}\right)^{l}=x^{k \cdot l}$.

The preceding two lemmas form the basis of an inductive proof of the two properties we desire and state explicitly in Theorem 1.1 below. The next two lemmas form the inductive parts of the proofs of each of the properties. Unfortunately, we seem to need both properties at each step in the induction, and the details become somewhat involved.

Lemma 1.6. Let $n \geqq 4$ and $x \geqq 2$. Assume that, for all $k \geqq 2$ and $l \geqq 0, f_{n-1}\left(f_{n-1}(x, k), l\right) \leqq f_{n-1}\left(x, f_{n-2}(k, l)\right)$ and further that for all $m \geqq n$ and for all $y$ and $z$

$$
f_{n-2}\left[f_{m}(x, y), f_{m}(x, z)\right] \leqq f_{m}(x, y+z) .
$$

Then, for all $m \geqq n$ and for all $y$ and $z$ we have

$$
f_{n-1}\left[f_{m}(x, y), f_{m}(x, z)\right] \leqq f_{m}(x, y+z) .
$$


Proof by induction on $m$. First, let us note directly from the equations $f_{n}(x, 0)=1$ and $f_{n}(1, y)=1$ that the cases $y=0$ and $z=0$ are immediate. Hence we shall assume now that both $y$ and $z$ are positive.

Consider the case $n=m$.

$$
\begin{aligned}
f_{n-1}\left\{f_{n}(x, y), f_{n}(x, z)\right\} \\
\quad=f_{n-1}\left\{f_{n-1}\left[x, f_{n}(x, y-1)\right], f_{n}(x, z)\right\} \\
\quad \leqq f_{n-1}\left\{x, f_{n-2}\left[f_{n}(x, y-1), f_{n}(x, z)\right]\right\} \\
\quad \leqq f_{n-1}\left\{x, f_{n}(x,(y+z)-1)\right\} \\
=f_{n}(x, y+z) .
\end{aligned}
$$

Here we used the first hypothesis with $k=f_{n}(x, y-1)$ and $l=f_{n}(x, z)$ and then the second hypothesis, and, of course, monotonicity.

Now let us assume the result for $m-1$ and prove it for $m$. As in Lemma 1.4 we shall prove the result for pairs $(y, z)$ with $z \geqq y$ and leave the remaining case to the reader. We have already noted that the result holds for pairs $(0, z)$. Let us now induce on $y$ (still assuming the result for all pairs $(y, z)$ at $m-1)$. Assuming we have the result for all $z \geqq y-1$ at $m$, we now establish the result for all pairs $(y, z)$ with $z \geqq y$.

$$
\begin{aligned}
f_{n-1}\left\{f_{m}(x, y), f_{m}(x, z)\right\} \\
\quad=f_{n-1}\left\{f_{m-1}\left[x, f_{m}(x, y-1)\right], f_{m-1}\left[x, f_{m}(x, z-1)\right]\right\} \\
\quad \leqq f_{m-1}\left\{x, f_{m}(x, y-1)+f_{m}(x, z-1)\right\} \\
\quad \leqq f_{m-1}\left\{x, f_{2}\left[f_{m}(x, y-1)+1, f_{m}(x, z-1)\right]\right\} \\
\quad \leqq f_{m-1}\left\{x, f_{n-2}\left[f_{m}(x, y-1)+1, f_{m}(x, z-1)\right]\right\} \\
\quad \leqq f_{m-1}\left\{x, f_{n-2}\left[f_{m}(x, y), f_{m}(x, z-1)\right]\right\} \\
\quad \leqq f_{m}(x, y+z) .
\end{aligned}
$$

Here we have used, in order, the assumption of the lemma at $m-1$, the fact that $a+b \leqq(a+1) b$, monotonicity $\left(f_{2}(a, b) \leqq f_{n-2}(a, b)\right.$ and then $f_{m}(x, y-1)<\left(f_{m}(x, y)\right)$, the second hypothesis of the lemma, and monotonicity.

Lemma 1.7. Let $n \geqq 4, x \geqq 2, k \geqq 2$ and $l \geqq 0$. Assume that $f_{n-1}\left[f_{n}(x, y), f_{n}(x, z)\right] \leqq f_{n}(x, y+z)$ for all $y$ and $z$. Then

$$
f_{n}\left(f_{n}(x, k), l\right)<f_{n}\left(x, f_{n-1}(k, l)\right) .
$$

Proof by induction on $l$. If $l=0$, the lemma is immediate. Assuming it for $l$, we now establish the case $l+1$. 


$$
\begin{aligned}
f_{n}\left(f_{n}(x,\right. & , k), l+1) \\
& =f_{n-1}\left\{f_{n}(x, k), f_{n}\left[f_{n}(x, k), l\right]\right\} \\
& <f_{n-1}\left\{f_{n}(x, k), f_{n}\left[x, f_{n-1}(k, l)\right]\right\} \\
& \leqq f_{n}\left\{x, k+f_{n-1}(k, l)\right\} \\
& \leqq f_{n}\left\{x, f_{n-2}\left[k, f_{n-1}(k, l)\right]\right\}=f_{n}\left(x, f_{n-1}(k, l+1)\right) .
\end{aligned}
$$

Here we used the assumption of the lemma at $l$, the hypothesis with $y=k$ and $z=f_{n-1}(k, l)$, and monotonicity to see that $f_{2}\left(k, f_{n-1}(k, l)\right) \leqq$ $f_{n-2}\left(k, f_{n-1}(k, l)\right)$.

We may now put the preceding four lemmas together into an inductive proof of the following theorem, with which we conclude our preliminary discussion of the functions $f_{n}(x, y)$.

THEOREM 1.1. For all $n \geqq 3, x \geqq 2, k \geqq 2$ and $l \geqq 0 ; f_{n}\left(f_{n}(x, k), l\right) \leqq$ $f_{n}\left(x, f_{n-1}(k, l)\right)$. (In fact the inequality is strict if $n>3$.) Further, for all $y$ and $z$ and for all $m \geqq n, f_{n-1}\left(f_{m}(x, y), f_{m}(x, z)\right) \leqq f_{m}(x, y+z)$.

Proof by induction on $n$. Lemmas 1.4 and 1.5 establish the two statements for $n=3$. In the inductive step, we assume both statements for $n-1$, and establish the second statement for $n$ by Lemma 1.6. Then the first statement follows for $n$ by Lemma 1.7.

2. The classes $\mathscr{G}_{n}$ of one-argument functions. In this section, we shall make use of iteration and a particular set of pairing functions, both due to Robinson, in defining a sequence of classes of one-argument primitive recursive functions. Let us being by stating some definitions.

Definition 2.1. The function $f(x)$ is said to be defined from $g(x)$ by iteration (more precisely, "pure iteration with no parameter beginning at 0 ") if $f(0)=0$ and $f(x+1)=g(f(x))$. We shall sometimes write $f(x)=g^{x}(0)$. The function $f\left(x^{(n)}\right)$ is said to be defined by the substitution of $h_{1}\left(x^{(n)}\right), \cdots, h_{m}\left(x^{(n)}\right)$ into $g\left(y^{(m)}\right)$ if

$$
f\left(x^{(n)}\right)=g\left(h_{1}\left(x^{(n)}\right), \cdots, h_{m}\left(x^{(n)}\right)\right) .
$$

Here either $m$ or $n$ is allowed to be 0 . We have used the notation $x^{(n)}$ for $x_{1}, \cdots, x_{n}$, which we shall employ freely below.

We shall make heavy use of the pairing functions $J, K$ and $L$ used in $[7, \S 4]$. These are defined as follows:

$$
\begin{aligned}
J(x, y) & =\left((x+y)^{2}+x\right)^{2}+y \\
K(z) & =E\left[z^{1 / 2}\right] \\
L(z) & =E(z) .
\end{aligned}
$$


Here $E(z)$ is the excess of $z$ over a square $\left(z-\left[z^{1 / 2}\right]^{2}\right)$, and $\left[z^{1 / 2}\right]$ is the largest integer not exceeding the square root of $z$. The important properties of these pairing functions are:

(1) $K(J(x, y))=x$ and $L(J(x, y))=y$ (but not necessarily $J(K(z), L(z))=z)$

(2) $J(0,0)=0$, hence $K(0)=L(0)=0$.

(3) If $L(z+1) \neq 0$ then $K(z+1)=K(z)$ and $L(z+1)=L(z)+1$ (even in those cases in which $z$ is not a value of $J(x, y)$ ).

The significance of (3) is the ease with which one can obtain all the pairs $(x, y), \cdots,(x, 0)$ from $J(x, y+1)=z$. Namely, merely list $(K(z-1), L(z-1)),(K(z-2), L(z-2), \cdots$ until you reach a pair of the form $(K(z-k), 0)$. This is used in reducing the number of arguments in recursive definitions in $[7, \S \S 3,4]$ (see Lemma 3.1 below).

Let us immediately generalize these functions $J, K$ and $L$ which establish a one-to-one correspondence between pairs of (nonnegative) integers and some integers. These new functions $J^{(n)}, M_{1}^{(n)}, \cdots, M_{n}^{(n)}$ will establish a correspondence between $n$-tuples and some integers, and will be used heavily in $\S 3$, especially in connection with the notion of "associate" defined there. The definitions are inductive and selfexplanatory (given our convention that $x^{(n)}$ means $x_{1}, \cdots, x_{n}$ ).

$$
\begin{aligned}
J^{(1)}(z) & =M_{1}^{(1)}(z)=z \\
J^{(n+1)}\left(x^{(n+1)}\right) & =J\left[J^{(n)}\left(x^{(n)}\right), x_{n+1}\right] \\
M_{i}^{(n+1)}(z) & =M_{i}^{(n)}(K(z)), \text { for all } 1 \leqq i \leqq n \\
M_{n+1}^{(n+1)}(z) & =L(z) .
\end{aligned}
$$

It is immediate from these definitions that the following generalizations of (1), (2), and (3) (which will be used in $\S 3$ ) hold:

(1') $M_{i}^{(n)}\left(J^{(n)}\left(x^{(n)}\right)=x_{i}\right.$ for all $1 \leqq i \leqq n$.

(2') $J^{(n)}(0, \cdots, 0)=0$, hence $M_{i}^{(n)}(0)=0$ for all $1 \leqq i \leqq n$.

$\left(3^{\prime}\right)$ If $M_{n}^{(n)}(z+1) \neq 0$ then $M_{i}^{(n)}(z+1)=M_{i}^{(n)}(z)$ for all $1 \leqq i \leqq$ $n-1$ and $M_{n}^{(n)}(z+1)=M_{n}^{(n)}(z)+1$ (even in those cases in which $z$ is not in the range of $\left.J^{(n)}\left(x^{(n)}\right)\right)$.

We shall use one further notation, this taken essentially from [6]. We shall write " $\langle f(x)=0 \rightarrow g(x) ; h(x)\rangle$ " for "either $g(x)$ if $f(x)=0$ or $h(x)$ otherwise". Given the functions $0^{x}=\langle x=0 \rightarrow 1 ; 0\rangle$ and $\operatorname{sgn}(x)=\langle x=0 \rightarrow 0 ; 1\rangle$, we can define $\langle f(x)=0 \rightarrow g(x) ; h(x)\rangle$ as $g(x) \cdot 0^{f(x)}+h(x) \cdot \operatorname{sgn}(f(x))$. Hence we may note that $\langle f(x)=0 \rightarrow$ $g(x) ; h(x)>$ is definable from $f(x), g(x), h(x), 0^{x}$ and $\operatorname{sgn}(x)$ by addition, multiplication and substitution of one-argument functions into oneargument functions.

Now we have the necessary background to define the classes $\mathscr{G}_{n}$ of one-argument functions. These will be defined to include the oneargument functions which we have just discussed and to be closed 
under the operations above as well. We make these statements more precise in Theorem 2.1 below.

DeFinition 2.2. We shall say the function $f\left(x^{(n)}, y\right)$ is defined from $g\left(x^{(n)}\right), h\left(x^{(n)}, y, z\right)$ and $j\left(x^{(n)}, y\right)$ by limited recursion (see [4]) if

$$
\begin{aligned}
f\left(x^{(n)}, 0\right) & =g\left(x^{(n)}\right) \\
f\left(x^{(n)}, y+1\right) & =h\left(x^{(n)}, y, f\left(x^{(n)}, y\right)\right) \\
f\left(x^{(n)}, y\right) & \leqq j\left(x^{(n)}, y\right) .
\end{aligned}
$$

We shall say that the function $f(x)$ is defined from $h(x)$ and $j(x)$ by limited iteration if $f(x)=h^{x}(0)$ and $f(x) \leqq j(x)$.

Definition 2.3. For each $n \geqq 2, \mathscr{G}_{n}$ is the smallest class of oneargument functions containing $S(x), E(x),\left[x^{1 / 2}\right]$ and $f_{n}(x, x)$ which is closed under addition $(f(x)=g(x)+h(x))$, multiplication $(f(x)=g(x) \cdot h(x))$, composition of one-argument functions $(f(x)=h(g(x)))$ and limited iteration.

THEOREM 2.1. Each class $\mathscr{G}_{n}$ contains

(i) the identity function $I(x)=x$,

(ii) the constant function $k(x)$ for each $k \geqq 0$,

(iii) $K(x)$ and $L(x)\left(\right.$ in fact $M_{i}^{(n)}(x)$ for each $n \geqq 1$ and $\left.1 \leqq i \leqq n\right)$,

(iv) $\operatorname{sgn}(x)=\langle x=0 \rightarrow 0 ; 1\rangle$,

(v) $0^{x}=\langle x=0 \rightarrow 1 ; 0\rangle$, and

(vi) $f_{i}(x, x)$ for $0 \leqq i \leqq 2$.

Further, each $\mathscr{G}_{n}$ is closed under

(vii) pairing $\left(f(x)=J^{(n)}\left(f_{1}(x), \cdots, f_{n}(x)\right)\right.$, and

(viii) conditional expression $(f(x)=\langle g(x)=0 \rightarrow h(x) ; j(x)\rangle \quad$ ).

Proof.

(i ) $I(x)=S^{x}(0), I(x) \leqq S(x)$.

(ii) $0(x)=I^{x}(0), 0(x) \leqq I(x)$, and $k(x)=S^{k}(0(x))$ for each fixed integer $k$.

(iii) $K(x)=E\left[x^{1 / 2}\right], L(x)=E(x)$ and each $M_{i}^{(n)}(x)$ is obtained by composition from these.

(iv) $\operatorname{sgn}(x)=1^{x}(0), \operatorname{sgn}(x) \leqq 1(x)$

(v) $0^{x}=E\left\{S^{2}[2(x) \cdot \operatorname{sgn}(x)]\right\}$

(vi) $f_{0}(x, x)=S(x)$

$$
\begin{aligned}
& f_{1}(x, x)=I(x)+I(x) \\
& f_{2}(x, x)=I(x) \cdot I(x)
\end{aligned}
$$

(vii) $J(g(x), h(x))=\left[(g(x)+h(x))^{2}+g(x)\right]^{2}+h(x)$, obtained by addition and multiplication from $g(x)$ and $h(x)$ 
(viii) $\langle g(x)=0 \rightarrow h(x) ; j(x)\rangle=h(x) \cdot 0^{g(x)}+j(x) \cdot \operatorname{sgn}(g(x))$ obtained by composition, addition and multiplication.

THEOREM 2.2. For any $n \geqq 2$ and any $f(x)$ in $\mathscr{C}_{n}$, there is a positive integer $k$ such that, for every $x \geqq 2, f(x)<f_{n+1}(x, k)$.

Proof. The theorem holds for the basic functions of $\mathscr{G}_{n}$, namely $S(x)<f_{2}(x, 2) \leqq f_{n+1}(x, 2), E(x)<x=f_{n+1}(x, 1),\left[x^{1 / 2}\right]<x=f_{n+1}(x, 1)$ and $f_{n}(x, x)=f_{n+1}(x, 2)<f_{n+1}(x, 3)$. Now assume inductively that $g(x)<f_{n+1}(x, k)$ and $h(x)<f_{n+1}(x, l)$ for $k$ and $l$ both greater than 1 (without loss of generality). We shall show that the four operations in terms of which $\mathscr{G}_{n}$ is defined preserve the boundedness property. If $f(x)$ is defined by limited iteration the result is obvious. If $f(x)=$ $g(x)+h(x)$ or $g(x) \cdot h(x)$, then, since $x, k$ and $l$ are all at least 2 , we have $f(x)<f_{n+1}(x, k) \cdot f_{n+1}(x, l) \leqq f_{n+1}(x, k+l)$ by Lemma 1.4. Finally, if $f(x)=h(g(x))$, we use Theorem 1.1 to see that

$$
\begin{aligned}
f(x) & <f_{n+1}\left(f_{n+1}(x, k), l\right) \\
& \leqq f_{n+1}\left(x, f_{n}(k, l)\right)
\end{aligned}
$$

as desired.

DEFINITION 2.4. The function $f(x)$ majorizes $g(x)$ if there is an integer $k$ such that $f(x)>g(x)$ for all $x \geqq k$.

COROLlaRY. For every $n \geqq 2$, the function $f_{n+1}(x, x)$ majorizes every function in $\mathscr{G}_{n}$. Hence $\mathscr{G}_{n} \neq \mathscr{G}_{m}$ for $m \neq n$.

The next theorem tells us that iteration (unlimited) does not lead out of the union of the $\mathscr{G}_{n}$. This, in connection with a result of Robinson [7 Th. 3], shows that $\cup \mathscr{G}_{n}$ is the class of all one-argument primitive recursive functions. However, we shall obtain that corollary result a little differently at the end of the next section, so we do not state it explicitly here.

We precede the theorem with a lemma which we shall use in its proof.

LEMMA 2.1. For every $n \geqq 2$ and $k \geqq 1$ the function $h_{n, k}(x)=$ $f_{n}(k, x)$ is in $\mathscr{G}_{m}$ for every $m \geqq n$; hence, in particular it is in $\mathscr{G}_{n}$.

Proof by induction on $n$. For $n=2$, we merely note that $h_{2, k}(x)=$ $k(x) \cdot I(x)$. Let us assume that $h_{n, k}(x)$ is in $\mathscr{C}_{m}$ for all $m \geqq n$ and all $k$. Then define $h_{n+1, k}^{\prime}(x)$ by: 


$$
\begin{aligned}
h_{n+1, k}^{\prime}(0) & =0 \\
h_{n+1, k}^{\prime}(x+1) & =g_{n, k}\left(h_{n+1, k}^{\prime}(x)\right) \\
h_{n+1, k}^{\prime}(x) & \leqq f_{n+1}(k, x) \leqq f_{n+1}(k+x, k+x) \leqq f_{m+1}(k+x, k+x)
\end{aligned}
$$

where $g_{n, k}(x)=h_{n, k}(\langle x=0 \rightarrow 1 ; x\rangle)$, a function in $\mathscr{G}_{m}$ for every $m \geqq n$ by Theorem 2.1 (viii) and $f_{m+1}(k+x, k+x)$ is in $\mathscr{G}_{m+1}$ since it results from substituting $k(x)+I(x)$ into the basic function $f_{m+1}(x, x)$. Thus $h_{n+1, k}^{\prime}(x)$ is in $\mathscr{G}_{m}$ for every $m \geqq n+1$, and $h_{n+1, k}(x)=\left\langle h_{n+1, k}^{\prime}(x)=\right.$ $\left.0 \rightarrow 1 ; h_{n+1, k}^{\prime}(x)\right\rangle$, is also in $\mathscr{G}_{m}$ as desired.

THEOREM 2.3. If $f(x)$ is defined by iteration from $g(x)$, that is, $f(x)=g^{x}(0)$, and if $g(x)$ is in $\mathscr{G}_{n}$, then $f(x)$ is in $\mathscr{G}_{n+1}$.

Proof. Let $g(x)$ be less than $f_{n+1}(x, k)$, for all $x \geqq 2$ and some $k \geqq 2$ (by Theorem 2.2). We shall show that, for any $x \geqq 2$ and $y \geqq 0, g^{x}(y) \leqq f_{n+1}\left(\bar{y}, f_{n+1}(k, x)\right)$ where $\bar{y}$ is the maximum of $y$ and 2 . From this it will follow that $f(x)=g^{x}(0) \leqq f_{n+1}\left(2, f_{n+1}(k, x)\right)$, which is, by monotonicity, at most $f_{n+1}\left(k, f_{n+1}(k, x)\right)$. But $f_{n+1}(k, x)$ is a oneargument function $h(x)$ in $\mathscr{G}_{n+1}$ by the lemma. Our bounding function is then $h^{2}(x)$, again in $\mathscr{G}_{n+1}$. Thus it suffices to show that $g^{x}(y) \leqq$ $f_{n+1}\left(\bar{y}, f_{n+1}(k, x)\right)$. Here $g^{x}(y)$ is taken to mean the function defined by

$$
g^{0}(y)=y, g^{x+1}(y)=g\left(g^{x}(y)\right) .
$$

Now $g^{0}(y)=y \leqq f_{n+1}\left(\bar{y}, f_{n+1}(k, 0)\right)$, so let us establish our bound on $g^{x}(y)$ by induction on $x$. Assuming that $g^{x}(y) \leqq f_{n+1}\left(\bar{y}, f_{n+1}(k, x)\right)$ for a fixed value of $x$ and for all $y$, we have

$$
\begin{aligned}
g^{x+1}(y) & =g\left(g^{x}(y)\right) \\
& \left.\leqq f_{n+1} \overline{\left(g^{x}(y)\right.}, k\right) \\
& \leqq f_{n+1}\left[f_{n+1}\left(\bar{y}, f_{n+1}(k, x)\right), k\right] \\
& \leqq f_{n+1}\left[\bar{y}, f_{n}\left[f_{n+1}(k, x), k\right]\right] \\
& =f_{n+1}\left(\bar{y}, f_{n+1}(k, x+1)\right) .
\end{aligned}
$$

Here we have used the first part of Theorem 1.1 and then the second part of Theorem 1.1 having noted that $k=f_{n+1}(k, 1)$.

CoRollary. The union of the classes $\mathscr{G}_{n}, n \geqq 2$, is closed under addition, multiplication, composition of one-argument functions and iteration.

3. The classes $\mathscr{F}_{n}$ of primitive recursive functions. In this section we define classes $\mathscr{F}_{n}$ which are essentially the $\mathscr{F}^{n}$ introduced by Grzegorczyk (see the final paragraph of $\S 4$ ). We shall use results 
of Robinson to show that, for each $n \geqq 2, \mathscr{G}_{n}$ is the class of all oneargument functions in $\mathscr{F}_{n}$. In fact, we shall show that $\mathscr{F}_{n}$ is the class of all functions which have their one-argument "associates" under the pairing functions included in $\mathscr{G}_{n}$. We shall make this precise in Definition 3.2 and Theorem 3.4 below.

We begin by defining the classes $\mathscr{F}_{\pi}$.

DEFinition 3.1. For each $n \geqq 0$, let $\mathscr{F}_{n}$ be the smallest class of functions containing the successor function $S(x)$, the zero function $0(x)$, the identity functions $U_{i}^{n}\left(x_{1}, \cdots, x_{n}\right)=x_{i}$, and $f_{n}(x, y)$ which is closed under substitutions and limited recursion (see Definitions 2.1 and 2.2).

It is an immediate consequence of this definition and the monotonicity of the $f_{n}(x, y)$ that $f_{n}(x, y)$ can be defined by limited recursion in every class $\mathscr{F}_{m}$ where $m$ is at least as large as $n$. Hence, $\mathscr{F}_{n}$ is contained in $\mathscr{F}_{m}$ for every $m \geqq n \geqq 0$. That this containment is proper will follow from the relation between $\mathscr{F}_{n}$ and $\mathscr{G}_{n}$ to be established below. We now prove a theorem which, though it consists only in listing several functions in $\mathscr{F}_{n}$, establishes part of this relationship.

Theorem 3.1. The functions $x+y, x \cdot y, x-y,\left[x^{1 / 2}\right], E x$ and $f_{n}(x, x)$ are in $\mathscr{F}_{n}$ for every $n \geqq 2$. Hence $\mathscr{S}_{n} \subset \mathscr{F}_{n}$ for every $n \geqq 2$.

Proof. We define a sequence of functions in $\mathscr{F}_{n}$ which includes the desired functions. Each definition is either by substitution or limited recursion from functions already known to be in $\mathscr{F}_{n}$.

$$
\begin{aligned}
& \begin{array}{ll}
x+0=x & x \cdot 0=0
\end{array} \\
& x+(y+1)=S(x+y) \quad x \cdot(y+1)=x+(x \cdot y) \\
& x+y \leqq f_{n}(S(S(x)), y), \quad x \cdot y \leqq f_{n}(x, y) \text {, (by Lemma 1.3) } \\
& \text { (see Lemma 1.3); } \\
& P(0)=0 \quad x-0=I(x) \quad 0^{0}=1 \\
& P(x+1)=I(x)=U_{1}^{1}(x) \quad x-(y+1)=P(x-y) \quad 0^{x+1}=0\left(0^{x}\right) \\
& P(x) \leqq I(x) \quad x-y \leqq U_{1}^{2}(x, y) \quad 0^{x} \leqq S(x) \\
& x^{2}=I(x) \cdot I(x) f_{n}(x, x)=f_{n}(I(x), I(x)) E(x)=x-\left[x^{1 / 2}\right]^{2} \\
& {\left[0^{1 / 2}\right]=0} \\
& {\left[(x+1)^{1 / 2}\right]=\left[x^{1 / 2}\right]+0^{\left(\left[x^{1 / 2}\right]+1\right)^{2}-(x+1)}} \\
& {\left[x^{1 / 2}\right] \leqq I(x) \text {. }}
\end{aligned}
$$

All the basic functions of $\mathscr{G}_{n}$ are among these. Hence we need only show that $\mathscr{F}_{n}$ is closed under the operations used in defining $\mathscr{C}_{n}$ to complete the proof. But this is immediate since the addition and 
multiplication functions are in $\mathscr{F}_{n}$ and it is closed under substitution and limited recursion.

We wish to show that $\mathscr{G}_{n}$ is precisely the class of all one-argument functions in $\mathscr{F}_{n}$. In fact, we shall show that $\mathscr{F}_{n}$ is just the class of all functions which can be obtained from $\mathscr{G}_{n}$ by use of the pairing functions alone. A critical step in this process is the replacement of definition by limited recursion with definition by its special case, limited iteration. The replacement of primitive recursion by iteration was carried out in [7, $\S 3$ and 4] following earlier work by Péter. We modify that replacement slightly so that it will yield immediate bounds on the functions in question and thus apply to the limited recursions and iterations we are using. Lemma 3.1 follows [7] directly, but we include it here for completeness and for notational convenience. Lemma 3.2 differs slightly from Robinson's work; the difference is introduced so that the $f^{\prime}(z)$ we obtain can be expressed in terms of the original function for all values of $z$, thus keeping the bound on $f^{\prime}(z)$ for all $z$.

LEMMA 3.1. Let $f\left(x^{(n)}, y\right)$ be defined by primitive recursion from $g\left(x^{(n)}\right)$ and $h\left(x^{(n)}, y, z\right)$ :

$$
\begin{aligned}
f\left(x^{(n)}, 0\right) & =g\left(x^{(n)}\right) \\
f\left(x^{(n)}, y+1\right) & =h\left(x^{(n)}, y, f\left(x^{(n)}, y\right)\right) .
\end{aligned}
$$

Then $F(z)=f\left(M_{1}^{(n+1)}(z), \cdots, M_{n+1}^{(n+1)}(z)\right)$, and hence $f\left(x^{(n)}, y\right)=$ $F\left(J^{(n+1)}\left(x^{(n)}, y\right)\right)$, where $F(z)$ is defined by

$$
\begin{aligned}
F(0) & =g\left(0^{(n)}\right) \\
F(z+1) & =H(z, F(z))
\end{aligned}
$$

where

$$
\begin{array}{r}
H(z, w)=\left\langle M_{n+1}^{(n+1)}(z+1)=0 \rightarrow g\left[M_{1}^{(n+1)}(z+1), \cdots, M_{n}^{(n+1)}(z+1)\right] ;\right. \\
\left.h\left(M_{1}^{(n+1)}(z), \cdots, M_{n+1}^{(n+1)}(z), w\right)\right\rangle,
\end{array}
$$

Proof by induction on $z$. Since $M_{i}^{(n+1)} 0=0$, we have the desired result for $z=0$. Assuming the result for $z$, we consider $F(z+1)$. There are two cases according as $M_{n+1}^{(n+1)}(z+1)=0$ or not. In the former case the result is immediate by the definition of $f\left(x^{(n)}, 0\right)$ as $g\left(x^{(n)}\right)$. In the latter case the result follows by induction and an observation that, by property $\left(3^{\prime}\right)$ of the pairing functions, $M_{i}^{(n+1)}(z+1)=$ $M_{i}^{(n+1)}(z)$ for all $1 \leqq i \leqq n$ and $M_{n+1}^{(n+1)}(z+1)-1=M_{n+1}^{(n+1)}(z)$.

LemMA 3.2. Let $F(z)$ be defined by primitive recursion:

$$
\begin{aligned}
F(0) & =k \\
F(z+1) & =H(z, F(z)) .
\end{aligned}
$$


Then $f^{\prime}(z)=\langle z=0 \rightarrow 0 ; J(z, F(z))\rangle$ and hence $F(z)=\langle z=0 \rightarrow k$; $\left.L\left(f^{\prime}(z)\right)\right\rangle$ where $f^{\prime}(z)$ is defined by pure iteration, namely:

$$
\begin{aligned}
f^{\prime}(0) & =0 \\
f^{\prime}(z+1) & =J\left\{K\left(f^{\prime}(z)\right)+1, H^{\prime}\left[K\left(f^{\prime}(z)\right), L\left(f^{\prime}(z)\right)\right]\right\}
\end{aligned}
$$

with

$$
H^{\prime}(z, w)=\langle z=0 \rightarrow H(0, k) ; H(z, w)\rangle \text {. }
$$

Proof. First we define $F^{\prime}(z)$, a function which agrees with $F(z)$ except when $z=0$ :

$$
\begin{aligned}
F^{\prime}(0) & =0 \\
F^{\prime}(z+1) & =H^{\prime}\left(z, F^{\prime}(z)\right) .
\end{aligned}
$$

Then we note that $f^{\prime}(z)=J\left(z, F^{\prime}(z)\right)$ as desired.

These two lemmas combine into the following general theorem.

THEOREM 3.2. Let $f\left(x^{(n)}, y\right)$ be defined by primitive recursion from $g\left(x^{(n)}\right)$ and $h\left(x^{(n)}, y, z\right)$, where $n$ is any nonnegative integer (possibly zero). Then there is a function $f^{\prime}(z)$ such that

$$
f^{\prime}(z)=\left\langle z=0 \rightarrow 0 ; J\left(z, f\left(M_{1}^{(n+1)}(z), \cdots, M_{n+1}^{(n+1)}(z)\right)\right\rangle,\right.
$$

and hence

$$
f\left(x^{(n)}, y\right)=\left\langle J^{(n+1)}\left(x^{(n)}, y\right)=0 \rightarrow g\left(0^{(n)}\right) ; L f^{\prime}\left(J^{(n+1)}\left(x^{(n)}, y\right)\right)\right\rangle
$$

where $f^{\prime}(x)$ is defined by pure iteration, namely

$$
\begin{aligned}
f^{\prime}(0) & =0 \\
f^{\prime}(z+1) & =H^{\prime \prime}\left(f^{\prime}(z)\right)
\end{aligned}
$$

with

$$
\begin{aligned}
H^{\prime \prime}(w) & =J\left\{K(w)+1, H^{\prime}(K(w), L(w))\right\}, \\
H^{\prime}(z, w) & =\left\langle z=0 \rightarrow H\left(0, g\left(0^{(n)}\right) ; H(z, w)\right\rangle,\right. \\
H(z, w) & =\left\langle M_{n+1}^{(n+1)}(z+1)=0 \rightarrow g\left[M_{1}^{(n+1)}(z+1), \cdots, M_{n}^{(n+1)}(z+1)\right] ;\right. \\
& h\left(M_{1}^{(n+1)}(z), \cdots, M_{n+1}^{(n+1)}(z), w\right\rangle .
\end{aligned}
$$

Now we are prepared to prove the two theorems which relate the classes $\mathscr{F}_{n}$ and $\mathscr{G}_{n}$. We begin with the definition of an associate of a function.

Definition 3.2. The function $g\left(x^{(m)}\right)$ is called the m-argument associate of the function $f\left(x^{(n)}\right)$ if $g\left(M_{1}^{(m)}(x), \cdots, M_{m}^{(m)}(x)\right)=$ $f\left(M_{1}^{(n)}(x), \cdots, M_{n}^{(n)}(x)\right)$ for all $x$. 
Let us note two important facts about associates. First, if $g\left(x^{(m)}\right)$ is an associate of $f\left(x^{(n)}\right)$ it must be the function

$$
f\left(M_{1}^{(n)}\left(J^{(m)}\left(x^{(m)}\right)\right), \cdots, M_{n}^{(n)}\left(J^{(m)}\left(x^{(m)}\right)\right)\right) .
$$

Thus there is at most one $m$-argument associate of any given $n$ argument function. However, there need not be any. For example, the one-argument function $f(x)=x$ does not have a 2-argument associate. For, assuming that $g(x, y)$ is an associate, we have by the above that $g(x, y)=f(J(x, y))=\left((x+y)^{2}+x\right)^{2}+y$. But this function $g(x, y)$ does not satisfy the defining condition that, for every $x, g(K(x), L(x))=$ $f(x)$. In particular $f(1)=1$ but $K(1)=L(1)=0$, hence $g(K(1), L(1))=$ 0 ; in fact $g\left(K\left(y^{4}\right), L\left(y^{4}\right)\right)=0$ for all $y$ since $K\left(y^{4}\right)=L\left(y^{4}\right)=0$. (The reader may show easily that in general an $n$-argument function $f\left(x^{(n)}\right)$ will have an $m$-argument associate if and only if $f\left(M_{1}^{(n)}(x), \cdots, M_{n}^{(n)}(x)\right)=$ $f\left(M_{1}^{(n)}(y), \cdots, M_{n}^{(n)}(y)\right)$ whenever $M_{i}^{(m)}(x)=M_{i}^{(m)}(y)$ for all $1 \leqq i \leqq m$. However, we make no use of this fact here.)

We shall show, in the next two theorems, that $\mathscr{F}_{n}$ is precisely the class of all associates of functions in $\mathscr{G}_{n}$.

THEOREM 3.3. The class of all associates of functions in $\mathscr{G}_{n}$ is closed under substitutions and limited recursion. Further, any function defined by primitive recursion from functions with associates in $\mathscr{G}_{n}$ has an associate in $\mathscr{G}_{n+1}$.

Proof. First let us consider substitutions. Assume $g_{i}\left(x^{(m)}\right), 1 \leqq$ $i \leqq n$, and $h\left(x^{(n)}\right)$ are associates of functions $g_{i}^{*}(x)$ and $h^{*}(x)$ in $\mathscr{G}_{k}$. Then $f\left(x^{(m)}\right)=h\left(g_{1}\left(x^{(m)}\right), \cdots, g_{n}\left(x^{(m)}\right)\right)$ is an associate of $f^{*}(x)=$ $h^{*}\left(J^{(n)}\left(g_{1}^{*}(x), \cdots, g_{n}^{*}(x)\right)\right)$. But $f^{*}(x)$ is in $\mathscr{G}_{k}$ by Theorem 2.1 (vii) and closure under substitutions.

Now let us turn to recursion. Assume $g\left(x^{(n)}\right)$ and $h\left(x^{(n)}, y, z\right)$ are associates of functions $g^{*}(x)$ and $h^{*}(x)$ in $\mathscr{G}_{k}$, and let $f\left(x^{(n)}, y\right)$ be defined from $g\left(x^{(n)}\right)$ and $h\left(x^{(n)}, y, z\right)$ by primitive recursion. By Theorem 3.2, $f^{*}(x)=f\left(M_{1}^{(n+1)}(x), \cdots, M_{n+1}^{(n+1)}(x)\right)=\left\langle x=0 \rightarrow g\left(0^{(n)}\right) ; L\left(f^{\prime}(x)\right)\right\rangle$, where the function $f^{\prime}(x)$ is defined by pure iteration from $H^{\prime \prime}(w)$ as described in the theorem. But $H^{\prime \prime}(w)$ is in $\mathscr{G}_{k}$, as we now show. It will be in $\mathscr{G}_{k}$ if $H^{\prime}(K(w), L(w))$ is, by the closure of $\mathscr{G}_{k}$ under conditional expression. For the same reason, $H^{\prime}(K(w), L(w))$ will be in $\mathscr{G}_{k}$ if $H(K(w), L(w))$ is. But this last function is precisely $\left\langle M_{n+1}^{(n+1)}(K(w)+\right.$ $\left.1)=0 \rightarrow g^{*}(K(K(w)+1)) ; h^{*}(w)\right\rangle$ which is in $\mathscr{G}_{k}$. Hence $H^{\prime \prime}(w)$ is in $\mathscr{G}_{k}$ so that an application of Theorem 2.3 shows that $f^{\prime}(x)$ is in $\mathscr{G}_{k+1}$. But then $f^{*}(x)$ is also in $\mathscr{G}_{k+1}$ as desired.

We have now only to show closure of the associates of $\mathscr{G}_{k}$ under limited recursion. To do so, we reason as follows. If, in fact, $f^{\prime}(x)$ 
is bounded by some function in $\mathscr{G}_{k}$, then $f^{\prime}(x)$ and hence $f^{*}(x)$ will also be in $\mathscr{G}_{k}$. But exactly this is the case if $f\left(x^{(n)}, y\right)$ is defined by limited recursion with a bounding function with an associate, say $j^{*}(x)$, in $\mathscr{G}_{k}$. For then $f^{\prime}(x) \leqq J\left(x, j^{*}(x)\right)$. This completes the proof of the theorem.

THEOREM 3.4. For every $n \geqq 2$, the class $\mathscr{F}_{n}$ is the class of all associates of all functions in $\mathscr{G}_{n}$.

Proof. First, we have $\mathscr{G}_{n} \subset \mathscr{F}_{n}$ by Theorem 3.1. But every associate of any $f(x)$ in $\mathscr{G}_{n}$ is obtained by substituting $J^{(k)}\left(x^{(k)}\right)$ into $f(x)$ by the remarks following the definition of asssociate. Since $\mathscr{F}_{n}$ is closed under substitutions by definition, all these associates are in $\mathscr{F}_{n}$. To prove the converse, we note that, by Theorem 3.3, it suffices to show that the basic functions of $\mathscr{F}_{n}$ are associates of functions in $\mathscr{G}_{n}$. This is obvious for all the basic functions except $f_{n}(x, y)$, and we shall prove that $f_{n}(x, y)$ has an associate in $\mathscr{G}_{n}$ by induction on $n$. We first observe that the function $K(x) \cdot L(x)$ is an associate of $f_{2}(x, y)$ which is in $\mathscr{G}_{2}$, so that the result holds for $n=2$. The inductive step follows from Theorem 3.3 since $f_{n+1}(x, y)$ is defined from $1(x)$ and $h(x, y, z)=f_{n}\left(U_{1}^{3}(x, y, z), U_{3}^{3}(x, y, z)\right)$ by primitive recursion.

We conclude this section with three corollaries which spell out the relationship of the classes $\mathscr{G}_{n}, \mathscr{F}_{n}$, and the class of all primitive recursive functions.

COROLlary. For every $n \geqq 2, \mathscr{G}_{n}$ is precisely the class of all one variable functions in $\mathscr{F}_{n}$.

Proof. $\mathscr{G}_{n}$ is the class of all one-variable associates of functions in $\mathscr{G}_{n}$.

COROLlaRY. For every $n \geqq 2, \mathscr{G}_{n}$ is properly contained in $\mathscr{G}_{n+1}$. For every $n \geqq 0, \mathscr{F}_{n}$ is properly contained in $\mathscr{G}_{n+1}$.

Proof. As commented immediately after the definition of the classes $\mathscr{F}_{n}$, each $\mathscr{F}_{n}$ is contained in $\mathscr{F}_{n+1}$. But, by the corollary to Theorem 2.2, $f_{n+1}(x, x)$ is in $\mathscr{F}_{n+1}$ but not $\mathscr{F}_{n}$.

COROLlaRY. The union of the increasing sequence of classes $\mathscr{F}_{n}$ is the class of all primitive recursive functions.

Proof. The primitive recursive functions can be characterized (see, for example, [3, p. 49]) as the smallest class of functions containing 
$S(x), 0(x)$ and $U_{\imath}^{n}\left(x_{1}, \cdots, x_{n}\right)$ which is closed under substitutions and primitive recursion. The very definition of the $\mathscr{F}_{n}$ provides containment of these basic functions and also closure under substitutions. Closure of the union under primitive recursion is provided by Theorems 3.3 and 3.4 .

4. Equality of $\mathscr{F}_{n}$ and $\mathscr{E}^{n}$. In this section we shall show that $\mathscr{F}_{n}=\mathscr{E}^{n}$ for every $n \geqq 0$. We begin with Grzegorczyk's definition of of $g_{n}(x, y)$ and $\mathscr{E}^{n}$.

Definition 4.1. We define $g_{n}(x, y)$ by setting

$$
\left.\begin{array}{rl}
g_{0}(x, y) & =x+1 \\
g_{1}(x, y) & =x+y \\
g_{2}(x, y) & =(x+1)(y+1) \\
g_{n+1}(x, 0) & =g_{n}(x+1, x+1) \\
g_{n+1}(x, y+1) & =g_{n+1}\left(g_{n+1}(x, y), y\right)
\end{array}\right\} n \geqq 2 .
$$

Then we define $\mathscr{E}^{n}$ as the smallest class of functions containing $S(x), 0(x), U_{i}^{n}\left(x_{1}, \cdots, x_{n}\right)$ and $g_{n}(x, y)$ which is closed under substitutions and limited recursion.

We have written $g_{n}(x, y)$ for the function which Grzegorczyk denoted by $f_{n}(y, x)$, and have trivially modified the list of basic functions of $\mathscr{E}^{n}$ (Grzegorczyk observed that it was sufficient to take $S(x), U_{1}^{2}(x, y)$ and $U_{2}^{2}(x, y)$ as basic since $0(x)$ and $U_{\imath}^{n}\left(x_{1}, \cdots, x_{n}\right)$ could be defined from these by substitutions).

Let us include for reference a list of monotonicity properties of the $g_{n}(x, y)$ which were established in $[4, \mathrm{p} .28]$. We shall use these frequently below in relating $f_{n}(x, y)$ to $g_{n}(x, y)$, and refer to them simply as "[4, p. 28]".

(1) $g_{n}(x, y)>x$ for $n \geqq 2$

(2) $g_{n}(x, y+1)>g_{n}(x, y)$ for $n \geqq 1$

(3) $g_{n}(x+1, y)>g_{n}(x, y)$ for $n \geqq 0$.

Each inequality is easily proved from the definitions.

Now let us prove a lemma to be used in relating $\mathscr{F}_{n}$ and $\mathscr{E}^{n}$. The first assertion of the lemma was noted and used by Grzegorczyk in the case $n=3[4, \mathrm{p} .29]$ and by Axt for all $n \geqq 3[2, \mathrm{p}$. $58]$.

Lemma 4.1. For every $n \geqq 3, g_{n}(x, y)=h_{n}\left(x, 2^{y}\right)$ where $h_{n}(x, y)$ is defined by 


$$
\begin{aligned}
h_{n}(x, 0) & =x \\
h_{n}(x, y+1) & =g_{n-1}\left(h_{n}(x, y)+1, h_{n}(x, y)+1\right) .
\end{aligned}
$$

Further, $f_{n}(x, y)<g_{n}(x, y)$ for every $n \geqq 2, x, y \geqq 0$.

Proof. A completely straightforward induction on $z$ establishes the identity $h_{n}\left(h_{n}(x, y), z\right)=h_{n}(x, y+z)$ for all $x, y$ and $z$. Then another straightforward induction on $y$ establishes that $g_{n}(x, y)=$ $h_{n}\left(x, 2^{y}\right)$ completing the first part of the lemma. Turning to the second assertion, we observe that $f_{2}(x, y)<g_{2}(x, y)$ is immediate. Let us assume now that $n \geqq 3$. In case $x=0$, we have $f_{n}(0,0)=1<g_{n-1}(1,1)=$ $g_{n}(0,0)$, by [4, p. 28]. Further $f_{n}(0, y+1) \leqq 1<g_{n}(0, y+1)$ by another application of $\left[4\right.$, p. 28]. In case $x=1$, we have $f_{n}(1, y)=$ $1<g_{n-1}(1,1)=g_{n}(0,0)<g_{n}(1, y)$ by $[4$, p. 28]. Thus we need to establish $f_{n}(x, y) \leqq g_{n}(x, y)$ only for $n \geqq 3, x \geqq 2$. In these cases we shall in fact show that $f_{n}(x, y)<h_{n}(x, y)<g_{n}(x, y)$. That $h_{n}(x, y)<g_{n}(x, y)$ follows immediately from the definition of $h_{n}(x, y)$, the property ([4, p. 28]) that $g_{n-1}(x, y)>x$ and the first part of this lemma. To see that $f_{n}(x, y)<h_{n}(x, y)$ we proceed inductively on $y$ assuming that we already have $f_{n-1}(x, y)<g_{n-1}(x, y)$. We begin by noting that $h_{n}(x, y) \geqq x$ by induction on $y$. Now $f_{n}(x, 0)=1<h_{n}(x, 0)$, and then

$$
\begin{aligned}
f_{n}(x, y+1) & =f_{n-1}\left(x, f_{n}(x, y)\right) \\
& <f_{n-1}\left(x, h_{n}(x, y)\right) \\
& <g_{n-1}\left(x, h_{n}(x, y)\right) \\
& <g_{n-1}\left(h_{n}(x, y)+1, h_{n}(x, y)+1\right) \\
& =h_{n}(x, y+1) .
\end{aligned}
$$

This completes our proof.

Now we establish the equality of $\mathscr{E}^{n}$ and $\mathscr{F}_{n}$.

THEOREM 4.1. For every $n \geqq 0, \mathscr{F}_{n}=\mathscr{E}^{n}$.

Proof. For $n=0,1$ the two classes have identical definitions. For $n \geqq 2$, to establish equality it will suffice to show that $f_{n}(x, y)$ is in $\mathscr{E}^{n}$ and that $g_{n}(x, y)$ is in $\mathscr{F}_{n}$. But, by the preceding lemma, $f_{n}(x, y)<g_{n}(x, y)$ for all $n \geqq 2$, and this allows us to define $f_{n}(x, y)$ by limited recursion in $\mathscr{E}^{n}$. Namely, $f_{2}(x, y)$ is defined by primitive recursion from $f_{1}(x, y)$, which we know to be in $\mathscr{E}^{1} \subseteq \mathscr{E}^{2}$. (That $\mathscr{E}^{n} \subseteq \mathscr{E}^{n+1}$ is proved in [4, pp. 33-4]). Since $f_{2}(x, y)$ is bounded by a function in $\mathscr{E}^{2}, f_{2}(x, y)$ is then in $\mathscr{E}^{2}$. Assuming $f_{n}(x, y)$ is in $\mathscr{E}^{n}$, we find that $f_{n+1}(x, y)$ is in $\mathscr{E}^{n+1}$ by the same argument. Now we know that $\mathscr{F}_{n} \subseteq \mathscr{E}^{n}$ and need only to define $g_{n}(x, y)$ within $\mathscr{F}_{n}$ to complete the proof. We shall proceed by induction on $n$, keginning at 
$n=2$ where $g_{2}(x, y)=f_{2}(x+1, y+1)$, a function in $\mathscr{F}_{n}$. Assume the result holds for $n$. By Theorems 3.3 and 3.4 we see that $h_{n+1}(x, y)$ is in $\mathscr{F}_{n+1}$ since it is defined by primitive recursion from functions in $\mathscr{F}_{n}$; namely $I(x)$ and $g_{n}\left(U_{3}^{3}(x, y, z)+1, U_{3}^{3}(x, y, z)+1\right)$. But then $g_{n+1}(x, y)=h_{n+1}\left(x, 2^{y}\right)$ is in $\mathscr{F}_{n+1}$ since $2^{y}=f_{3}(2, y)$ is in $\mathscr{F}_{n+1}$ for all $n \geqq 2$.

Technically, Grzegorczyk did not pose his question about the classes $\mathscr{F}_{n}$ based upon the functions $f_{n}(x, y)$. Instead he asked about classes $\mathscr{F}^{n}$ whose definition is obtained from that of $\mathscr{F}_{n}$ by replacing $f_{n}(x, y)$ by $f_{n}^{\prime}(x, y)$. The definition of these functions $f_{n}^{\prime}(x, y)$ is in turn obtained from that of $f_{n}(x, y)$ by deleting the special definition of $f_{2}(x, y)$ and letting the general case cover that as well. Namely, (except for the fact that we are consistently reversing the order of his variables) Grzegorczyk defined $f_{n}^{\prime}(x, y)$ as

$$
\left.\begin{array}{rl}
f_{0}^{\prime}(x, y) & =x+1 \\
f_{1}^{\prime}(x, y) & =x+y \\
f_{n+1}^{\prime}(x, 0) & =1 \\
f_{n+1}^{\prime}(x, y+1) & =f_{n}^{\prime}\left(x, f_{n+1}^{\prime}(x, y)\right)
\end{array}\right\} \text { for all } n \geqq 1 .
$$

This makes $f_{2}^{\prime}(x, y)=x \cdot y+1, f_{3}^{\prime}(x, y)=x^{y}+\cdots+x^{1}+1=\left(x^{y+1}-1\right) /(x-1)$, and $f_{n}^{\prime}(x, y)$ for larger $n$ relatively inexpressible. It is easily seen that $f_{n}(x, y) \leqq f_{n}^{\prime}(x, y)$ for all $n \geqq 2$ and all $x$ and $y$. We leave the details of the proof, which by now should suggest themselves immediately, to the reader. From this it follows that $f_{n+1}^{\prime}(x, x)$ majorizes every oneargument function in $\mathscr{F}_{n}$. The containment of $\mathscr{F}_{n}$ in $\mathscr{F}^{n}$ follows since $f_{n}(x, y)$ is definable within $\mathscr{F}^{n}$ by limited recursion. But then $\mathscr{F}_{n}=\mathscr{F}^{n}$ since $f_{n}^{\prime}(x, y)$ is in $\mathscr{F}_{n}$ by induction on $n$ using Theorems 3.3 and 3.4 and the fact that $f_{n+1}^{\prime}(x, y)$ is defined from $f_{n}^{\prime}(x, y)$ by recursion.

\section{Bibliography}

1. Wilhelm Ackermann, Zum Hilbertschen Aufbau der reellen Zahlen, Math. Annalen 99 (1928), 118-133.

2. Paul Axt, Enumeration and the Grzegorczyk hierarchy, Zeitscharift fur math. Logik und Grundlagen der Math. 9 (1963), 53-65.

3. Martin Davis, Computability and Unsolvability, McGraw-Hill, New York, N.Y., (1958), $25+210 \mathrm{pp}$.

4. Andrej Grzegorczyk, Some classes of recursive functions, Rozprawy Matematyczne, Warsaw, (1953), $46 \mathrm{pp}$.

5. S. C. Kleene, Introduction to metamathematics, Van Nostrand, Princeton, N. J., (1952), $10+550 \mathrm{pp}$.

6. John McCarthy, Recursive functions of symbolic expressions and their computation by machine, Part I, Communications of the Assoc. for Computing Machinery 3 (1960), 184-194.

7. R. M. Robinson, Primitive recursive functions, Bull. Amer. Math. Soc. 53 (1947), 925-942. 


\section{PACIFIC JOURNAL OF MATHEMATICS}

\section{EDITORS}

\author{
H. Samelson \\ Stanford University \\ Stanford, California \\ R. M. Blumenthal \\ University of Washington \\ Seattle, Washington 98105
}

\author{
J. Dugundu \\ University of Southern California \\ Los Angeles, California 90007 \\ *Richard Arens \\ University of California \\ Los Angeles, California 90024
}

\section{ASSOCIATE EDITORS}
E. F. BeCKENBACH
B. H. NeUMANN
F. WOLF
K. YosidA

\section{SUPPORTING INSTITUTIONS}

\author{
UNIVERSITY OF BRITISH COLUMBIA \\ CALIFORNIA INSTITUTE OF TECHNOLOGY \\ UNIVERSITY OF CALIFORNIA \\ MONTANA STATE UNIVERSITY \\ UNIVERSITY OF NEVADA \\ NEW MEXICO STATE UNIVERSITY \\ OREGON STATE UNIVERSITY \\ UNIVERSITY OF OREGON \\ OSAKA UNIVERSITY \\ UNIVERSITY OF SOUTHERN CALIFORNIA
}

\author{
STANFORD UNIVERSITY \\ UNIVERSITY OF TOKYO \\ UNIVERSITY OF UTAH \\ WASHINGTON STATE UNIVERSITY \\ UNIVERSITY OF WASHINGTON \\ * * * * \\ AMERICAN MATHEMATICAL SOCIETY \\ CALIFORNIA RESEARCH CORPORATION \\ SPACE TECHNOLOGY LABORATORIES \\ NAVAL ORDNANCE TEST STATION
}

Mathematical papers intended for publication in the Pacific Journal of Mathematics should by typewritten (double spaced). The first paragraph or two must be capable of being used separately as a synopsis of the entire paper. It should not contain references to the bibliography. No separate author's resumé is required. Manuscripts may be sent to any one of the four editors. All other communications to the editors should be addressed to the managing editor, Richard Arens, at the University of California, Los Angeles, California 90024.

50 reprints per author of each article are furnished free of charge; additional copies may be obtained at cost in multiples of 50 .

The Pacific Journal of Mathematics is published quarterly, in March, June, September, and December. Effective with Volume 13 the price per volume (4 numbers) is $\$ 18.00$; single issues, $\$ 5.00$. Special price for current issues to individual faculty members of supporting institutions and to individual members of the American Mathematical Society: $\$ 8.00$ per volume; single issues $\$ 2.50$. Back numbers are available.

Subscriptions, orders for back numbers, and changes of address should be sent to Pacific Journal of Mathematics, 103 Highland Boulevard, Berkeley 8, California.

Printed at Kokusai Bunken Insatsusha (International Academic Printing Co., Ltd.), No. 6, 2-chome, Fujimi-cho, Chiyoda-ku, Tokyo, Japan.

PUBLISHED BY PACIFIC JOURNAL OF MATHEMATICS, A NON-PROFIT CORPORATION

The Supporting Institutions listed above contribute to the cost of publication of this Journal, but they are not owners or publishers and have no responsibility for its content or policies.

* Basil Gordon, Acting Managing Editor until February 1, 1966. 


\section{Pacific Journal of Mathematics}

\section{Vol. 15, No. $3 \quad$ November, 1965}

David R. Arterburn and Robert James Whitley, Projections in the space of

bounded linear operators .................................

Robert McCallum Blumenthal, Joram Lindenstrauss and Robert Ralph Phelps,

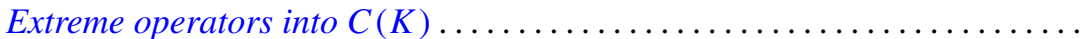

L. Carlitz, A note on multiple exponential sums ................... 757

Joseph A. Cima, A nonnormal Blaschke-quotient .................... 767

Paul Civin and Bertram Yood, Lie and Jordan structures in Banach algebras . . . 775

Luther Elic Claborn, Dedekind domains: Overrings and semi-prime

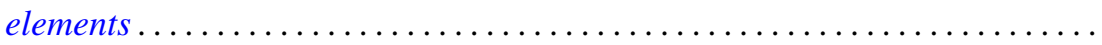

799

Luther Elic Claborn, Note generalizing a result of Samuel's .............. 805

George Bernard Dantzig, E. Eisenberg and Richard Warren Cottle, Symmetric

dual nonlinear programs ................................... 809

Philip J. Davis, Simple quadratures in the complex plane ............... 813

Edward Richard Fadell, On a coincidence theorem of F. B. Fuller ............ 825

Delbert Ray Fulkerson and Oliver Gross, Incidence matrices and interval

graphs ........................................ 835

Larry Charles Grove, Tensor products over $H^{*}$-algebras ..................

Deborah Tepper Haimo, $L^{2}$ expansions in terms of generalized heat polynomials

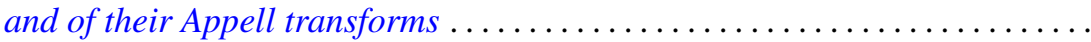

I. Martin (Irving) Isaacs and Donald Steven Passman, A chardcterization of groups in terms of the degrees of their characters ..........

Donald Gordon James, Integral invariants for vectors over local fields........ 905

Fred Krakowski, A remark on the lemma of Gauss ................... 917

Marvin David Marcus and H. Minc, A subdeterminant inequality ........... 921

Kevin Mor McCrimmon, Norms and noncommutative Jordan algebras ........ 925

Donald Earl Myers, Topologies for Laplace transform spaces ............... 957

Olav Njstad, On some classes of nearly open sets .................... 961

Milton Philip Olson, A characterization of conditional probability ........... 971

Barbara Osofsky, A counter-example to a lemma of Skornjakov .............. 985

Sidney Charles Port, Ratio limit theorems for Markov chains ............... 989

George A. Reid, A generalisation of $W^{*}$-algebras ...................... 1019

Robert Wells Ritchie, Classes of recursive functions based on Ackermann's

function ........................................... 1027

Thomas Lawrence Sherman, Properties of solutions of $n$th order linear

differential equations ........................................ 1045

Ernst Snapper, Inflation and deflation for all dimensions . .............. 1061

Kondagunta Sundaresan, On the strict and uniform convexity of certain Banach

spaces............................................. 1083

Frank J. Wagner, Maximal convex filters in a locally convex space .......... 1087

Joseph Albert Wolf, Translation-invariant function algebras on compact 